\title{
Changes in retinal nerve fiber layer thickness measurements in response to a trifocal intraocular lens implantation
}

\author{
Javier García-Bella ${ }^{1,2,3} \cdot$ Paula Talavero-González ${ }^{4} \cdot$ Jesús Carballo-Álvarez $^{5}$ - Juan C. Sanz-Fernández ${ }^{5}$. \\ José M. Vázquez-Moliní ${ }^{5}$ Julián García-Feijóo ${ }^{1,2,3}$ • José M. Martínez-de-la-Casa ${ }^{1,2,3}$
}

Received: 21 November 2017 / Revised: 26 April 2018 / Accepted: 3 May 2018 / Published online: 11 June 2018

(c) The Royal College of Ophthalmologists 2018

\begin{abstract}
Aims To determine the changes produced on the peripapillary retinal nerve fiber layer (RNFL) thickness, measured by an spectral-domain optical coherence tomography (OCT), after the implantation of a trifocal diffractive intraocular lens (IOL) and compare them with the variations produced by a monofocal IOL implantation.

Methods A prospective, double masked study in which 50 eyes belonging to 50 patients with bilateral cataract were enrolled. Sequentially, the first 25 patients were bilaterally implanted with the trifocal diffractive IOL AT LISA ${ }^{\circledast}$ Tri 839 MP and the following 25 patients with the monofocal IOL CT ASPHINA ${ }^{\circledR} 409$ M/MP. RNFL thickness measurements were performed by Cirrus $\mathrm{HD}^{\circledR}$-OCT before and six months after the second eye surgery.

Results Mean patient age was $69.5 \pm 6.1$ years. In response to surgery, the average RNFL thickness increased $7.29 \pm 10.51$ $\mu \mathrm{m}$ at the AT LISA ${ }^{\circledR}$ Tri group and $1.96 \pm 2.90 \mu \mathrm{m}$ at the monofocal IOL group from the baseline $(p=0.017)$. Statistically significant differences in thickness variations were also detected in the superior sector $\left(7.86 \pm 7.70 \mu \mathrm{m}\right.$ at the AT LISA $^{\circledR}$ Tri $^{2}$ group vs $1.73 \pm 5.74 \mu \mathrm{m}$ at the monofocal group). Statistically significant differences between the pre and the postsurgery measurements were found in all sectors except the inferior one with the trifocal IOL and in two of the studied sectors (average and temporal) with the monofocal IOL.

Conclusions The IOL implantation produce changes in the RNFL thickness, which are larger in case of a trifocal diffractive IOL.
\end{abstract}

\section{Introduction}

Currently, the trifocal intraocular lenses (IOLs) are increasingly used to achieve a satisfactory vision levels at far, near or intermediate vision without a following optical correction needed.

Javier García-Bella

Javier.garciabll@gmail.com

1 Servicio de Oftalmología, Hospital Clínico San Carlos, Madrid, Spain

2 Departamento de Oftalmología, Facultad de Medicina, Universidad Complutense de Madrid, Madrid, Spain

3 Instituto de Investigación Sanitaria del Hospital Clínico San Carlos (IdISSC), Madrid, Spain

4 Servicio de Oftalmología, Fundación Jiménez Díaz, Madrid, Spain

5 Facultad de Óptica, Universidad Complutense de Madrid, Madrid, Spain
The IOL AT LISA ${ }^{\circledR}$ Tri 839 MP (Carl Zeiss Meditec, Jena, Germany) is a trifocal diffractive lens, which produce an addition of +3.33 diopters (D) in the near vision and $+1.66 \mathrm{D}$ in the intermediate one. It has been designed as a plate with four haptics, a 0 degrees angulation and its square edge design also offer a 360 degree anti-posterior capsular opacification double barrier for double posterior capsular opacification protection. It has been commercialized in a dioptric range between 0.00 and $+32.00 \mathrm{D}$ in 0.50 D steps.

The use of optical coherence tomography (OCT) to examine the retinal nerve fiber layer (RNFL) has become essential in daily clinical practice. Several studies have shown the OCT clinical usefulness to study many retinal and optic nerve diseases [1,2], along with its excellent reproducibility [3].

In addition, recent studies have shown that following cataract extraction and the implantation of a monofocal IOL, the OCT measurements will be modified [4], which should be taken into account during the patients' follow-up 
visits. The possible changes produce by the frequent implantation of others IOLs still need to be investigated.

The present study was designed to determine if changes in the OCT peripapillary RNFL thickness measurements produced in response to cataract surgery with an IOL implantation, differ according to whether the implanted IOL is a monofocal or a trifocal one. This is important because if different types of IOL produce different changes in OCT measurements we must know it to make a correct follow-up of the patients depending on the type of the IOL implanted.

To the best of our knowledge, this is the first study to compare the monofocal versus the trifocal IOL implantation effects on OCT measurements.

\section{Methods}

The present experimental and prospective single-center study is adhered to the principles of the Helsinki Declaration of 1975. The study protocol was approved by the Institutional Review Board and all patients were informed and signed informed consent prior to inclusion.

The study sample included 50 eyes of 50 Caucasian subjects. Participants had been diagnosed with bilateral cataract in surgical range and a best-corrected distance (4 meters) visual acuity under 0.5 (Snellen scale). Further inclusion criteria were age older than 18 years and a presurgery refractive error of $\leq 5$ diopters of sphere (to increase the OCT scans accuracy) and $\leq 1.5$ diopters of corneal astigmatism. The cataracts were classified using the LOCS III system and the ones above N5,P3,C3 were excluded.

We excluded all patients presenting an uncontrolled systemic disease, cataracts as a result of trauma or medication, a prior or current ophthalmologic disease besides cataract or a previous retinal/optic disc pathologies such as glaucoma or other neuropathies.

All patients underwent cataract surgery using the phacoemulsification standard procedures with a Bausch and Lomb Stellaris ${ }^{\circledR}$ platform (Bausch\&Lomb Incorporated, Rochester, NY, USA) and an IOL implantation in the capsular bag. All surgical procedures were performed by the same surgeon (JMC). There were no intraoperative complications so there was no need to modify the original protocol. The different type of IOL implanted was assigned to patients in chronological consecutive order according to their inclusion date in the study. The first 25 patients were bilaterally implanted with the IOL AT LISA ${ }^{\circledR}$ Tri 839 MP (Carl Zeiss Meditec, Jena, Germany), which is a trifocal lens with two diffractive structures providing three visual foci. The following 25 patients bilaterally received the monofocal intraocular lens CT ASPHINA 409 M/MP (Carl Zeiss Meditec, Jena Germany). Only one eye (right) of each patient was included in the study.
In order to analyse the peripapillary RNFL thickness, spectral-domain OCT measurements were performed by Cirrus HD ${ }^{\circledR}$-OCT (Carl Zeiss Meditec, Jena, Germany) before surgery and six months after the second eye surgery to avoid any possible bias due to postsurgery inflammation. On the examination screen, we selected the mode "Optic Disc Cube $200 \times 200$ " to capture images and analyze the results. The measurements were repeated until the required quality level $(\geq 6 / 10)$ was achieved. All measurements were taken by the same researcher (JGB) who was not aware of the IOL type implanted in each subject.

As the groups could be not homogeneous, in both of them the presurgery RNFL measurements were subtracted to the postsurgery ones and the outcome result was the variable compared between the two groups. This way, differences between these new variables will be only ones due to the type of IOL implanted.

Thickness measurements are provided for each of the 5 sectors defined by this OCT instrument as: average thickness $(\mathrm{G})$, nasal $(\mathrm{N})$, temporal $(\mathrm{T})$, superior $(\mathrm{S})$, and inferior (I).

\section{Statistical analysis}

Statistical tests were performed using the program SPSS version 18.0 for Windows (SPSS Inc., Chicago, IL). The distribution of the different quantitative variables of the study was analyzed by the Kolmogorov-Smirnov test and afterwards, a parametric test was used for normally distributed variables (test for paired Student T) and a nonparametric one for non-normally distributed variables (test Wilcoxon). Quantitative variables were expressed by the corresponding mean and standard deviation (SD). Significance was set at $p<0.05$.

\section{Results}

The mean patient age was $70.3 \pm 6.0$ years in the IOL AT LISA $^{\circledR}$ Tri implanted group and $68.7 \pm 6.2$ years in the IOL CT ASPHINA $^{\circledR}$ group. 25 eyes of 25 patients were included in each group. No statistically significant differences between groups were found ( $p=0.431)$.

There were also no statistically significant differences between the men: women ratio (12:13 in the AT LISA ${ }^{\circledR}$ Tri group; $11: 14$ in the CT ASPHINA ${ }^{\circledR}$ group) $(p=0.327)$. All patients completed the follow-up visits and no OCT images were eliminated due to insufficient quality.

The cataracts included in the study allowed us to take the measurements required, always keeping a quality image level $\geq 6 / 10$. Mean image quality level before surgery was $6.75 \pm 0.63$ in ASPHINA $^{\circledR}$ group and $6.81 \pm 0.77$ in LISA $^{\circledR}$ Tri group. Postsurgery mean image quality level was 
Table 1 Peripapillary RNFL thickness measurements obtained for the IOL AT LISA ${ }^{\circledast}$ Tri group

\begin{tabular}{llll}
\hline & Presurgery & Postsurgery & $\mathrm{P}$ \\
\hline G & $79.67 \pm 15.21$ & $86.95 \pm 10.34$ & $0.005^{\mathrm{a}}$ \\
$\mathbf{N}$ & $59.95 \pm 15.09$ & $67.05 \pm 12.50$ & $0.048^{\mathrm{a}}$ \\
$\mathbf{S}$ & $95.52 \pm 23.85$ & $103.38 \pm 24.11$ & $<0.001^{\mathrm{a}}$ \\
$\mathbf{T}$ & $64.71 \pm 15.34$ & $71.48 \pm 9.12$ & $0.014^{\mathrm{a}}$ \\
$\mathbf{I}$ & $100.71 \pm 20.29$ & $105.10 \pm 16.11$ & 0.277 \\
\hline
\end{tabular}

Results expressed in microns (mean \pm standard deviation)

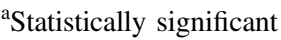

Table 2 Peripapillary RNFL thickness measurements obtained for the IOL CT ASPHINA ${ }^{\circledR}$ group

\begin{tabular}{llll}
\hline & Presurgery & Postsurgery & $P$ \\
\hline $\mathrm{G}$ & $100.35 \pm 6.44$ & $102.08 \pm 7.15$ & $0.008^{*}$ \\
$\mathrm{~N}$ & $79.31 \pm 7.68$ & $80.77 \pm 7.57$ & 0.074 \\
$\mathrm{~S}$ & $119.65 \pm 8.96$ & $121.38 \pm 11.05$ & 0.137 \\
$\mathrm{~T}$ & $79.15 \pm 6.73$ & $80.96 \pm 5,26$ & $0.032^{*}$ \\
$\mathrm{I}$ & $132.54 \pm 9.90$ & $132.77 \pm 10.99$ & 0.836 \\
\hline
\end{tabular}

Results expressed in microns (mean \pm standard deviation)

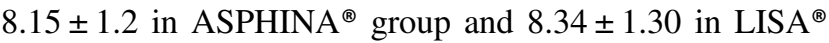
Tri group. No statistical differences were found between groups ( $p=0.24$ presurgery and $p=0.18$ postsurgery).

The best-corrected binocular levels of visual acuity (BCVA) before surgery were $0.75 \pm 0.18$ (LogMAR) in the IOL AT LISA ${ }^{\otimes}$ Tri 839 MP group and $0.73 \pm 0.23$ (LogMAR) in the IOL CT ASPHINA ${ }^{\circledR}$ group. No statistically significant differences between groups were found $(p=0.538)$.

Peripapillary RNFL thickness measurements obtained before and after surgery are provided in Table 1 for the IOL AT LISA ${ }^{\circledast}$ Tri group. Statistically differences between pre and postsurgery RNFL thickness values were observed in all analysed sectors except the inferior one.

Peripapillary RNFL thickness measurements obtained before and after surgery are provided in Table 2 for the IOL CT ASPHINA ${ }^{\circledast}$ group. Statistically differences between pre and postsurgery RNFL thickness values were observed in the average and temporal sectors.

Table 3 shows the comparison between the differences (postsurgery RNFL- presurgery RNFL measurements) found in both groups. Statistically significant differences were obtained in the average thickness and the superior sector. No statistically differences were found in the nasal sector although the $p$-value was very close to 0.05 . In addition, it can be observed that the RNFL thickness was particularly increased at the trifocal group versus the monofocal one.
Table 3 Differences (postsurgery RNFL-presurgery RNFL measure-ments) by sectors between the two study groups

\begin{tabular}{llll}
\hline & AT LISA Tri group & CT ASPHINA group & P \\
\hline Dif. G & $7.29 \pm 10.51$ & $1.96 \pm 2.90$ & $0.017^{\mathrm{a}}$ \\
Dif. N & $7.10 \pm 15.78$ & $0.77 \pm 4.47$ & 0.057 \\
Dif. S & $7.86 \pm 7.70$ & $1.73 \pm 5.74$ & $0.003^{\mathrm{a}}$ \\
Dif. T & $6.76 \pm 11.50$ & $2.27 \pm 4.57$ & 0.075 \\
Dif. I & $4.38 \pm 17.95$ & $0.62 \pm 6.25$ & 0.323 \\
\hline
\end{tabular}

Results expressed in microns (mean \pm standard deviation)

${ }^{\text {a }}$ Statistically significant

\section{Discussion}

Nowadays, the multifocal IOLs and especially the trifocal ones, are being frequently used to provide a very good quality and spectacle-free vision levels at far, near, and intermediate distance. In addition, the OCT has become a technique routinely used in ophthalmology clinics to examine the peripapillary RNFL thickness.

According to these facts, there is a need for knowledge on the OCT changes produced in response to this type of IOL implantation.

Previous studies had demonstrated the cataract effect on the OCT RNFL thickness measurements, which generally produce a low quality image due to light dispersal [5-7]. The IOL implantations produce a quality image improvement, which would result in a higher postsurgery RNFL thickness [8-10]. These findings have been demonstrated regardless of the IOL type implanted [4, 11, 12], but there are no studies in which a comparison between two different types of IOL had been realized.

In the present study, a higher postsurgery RNFL thickness was found in both groups, which agreed with other published studies. In the trifocal IOL group, the RNFL thickness increase was statistically significantly higher in all analyzed sectors but the inferior one. Previous studies with monofocal IOL was shown no changes in inferior sector but in this case the absence of variation in this sector could be producted by optical properties of the IOL.

To our knowledge there are no previous studies comparing the OCT measurement's changes produced in response to the implantation of different IOLs so we have no reported data to compare our results with.

As the first study to compare the variations induced by two different types of IOL, our findings are innovative and provide direction for future studies designed to address this issue.

The results obtained in this study indicate a significantly greater RNFL thickness increase, (measured by Cirrus ${ }^{\circledR}$ OCT) in the average thickness and the superior sectors following a trifocal IOL implantation than a monofocal one. 
Such results, especially the ones produced on average thickness, are of particular interest in daily clinical practice given their relevance in the study of glaucoma.

On the other hand, a study recently published by our team compares the effect of two different trifocal IOLs on the optical OCT measurements [13].

In the present study, OCT changes in the trifocal group were found on the same sectors as the ones observed in the previously mentioned study. The changes produced by a monofocal IOL it could also be compared in this study.

The peripapillary RNFL thickness differences produced by a multifocal IOL implantation compared to a monofocal one have not been detected in macular studies based on spectral-domain OCT or other OCT systems [14, 15].

Our findings indicate a significantly higher RNFL thickness increase, measured by Cirrus ${ }^{\circledR}$-OCT, in the average thickness and in the superior sectors after a trifocal IOL implantation than a monofocal one. Therefore, a higher RNFL thickness measurement found on a patient who had been previously implanted with a trifocal IOL, should be interpreted carefully because could be due to the lens optical variations produced on the OCT images.

Our novels findings need to be confirmed in future work although provide a starting point for further investigations into the possible impacts of these IOLs following their implant.

In conclusion, our findings indicate that cataract surgery with IOL implantation produce modifications in the peripapillary RNFL thickness measurements made by spectraldomain OCT and that these modifications vary according to the type of IOL implanted. These findings need confirmation in future studies.

\section{Summary}

\section{What was known before}

- Cataract surgery induces variations in measurements made by optical coherence tomography in macular thickness and retinal nerve fiber layer.

- These variations occur with different type of IOL.

\section{What this study adds}

- This is the first study that compares the alterations induce by two types of trifocal diffractive IOL with the alterations induce by a monofocal IOL in RNFL measurements made with spectral-domain OCT.

- The implantation of trifocal diffractive IOLs produces higher alterations in RNFL measurements made by spectral-domain optical coherence tomography than the implantation of monofocal IOL.

- This alterations occur mainly in average thickness and temporal sectors, so it has been considered in the followup of patients implanted with this type of IOL.

Acknowledgements Optical School, Complutense University of Madrid, Madrid, Spain.

\section{Compliance with ethical standards}

Conflict of interest The authors declare that they have no conflict of interest.

\section{References}

1. Carpintero P, Nubile M, Agnifili L, Toto L, Aharrh-Gnama A, Mastropasqua R, et al. Reproducibility and repeatability of Cirrus HD-OCT peripapillary retinal nerve fiber layer thickness measurements in young normal subjects. Ophthalmologica. 2012;227:139-45.

2. García-Martín E, Satue M, Fuertes I, Otin S, Alarcia R, Herrero $\mathrm{R}$, et al. Ability and reproducibility of Fourier-domain optical coherence tomography to detect retinal nerve fiber layer atrophy in Parkinson's disease. Ophthalmology. 2012; 119:2161-7.

3. Matlach J, Wagner M, Malzahn U, Göbel W. Repeatability of peripapillary retinal nerve fiber layer and inner retinal thickness among two spectral domain optical coherence tomography devices. Invest Ophthalmol Vis Sci. 2014;55:6536-46.

4. Bambo MP, García-Martin E, Otin S, Sancho E, Fuertes I, Herrero $\mathrm{R}$, et al. Influence of cataract surgery on repeatability and measurements of spectral domain optical coherence tomography. Br J Ophthalmol. 2014;98:52-8.

5. Van Velthoven MEJ, Van der Linden MH, De Smet MD, Faber DJ, Verbraak FD. Influence of cataract on optical coherence tomography image quality and retinal thickness. Br J Ophthalmol. 2006;90:1259-62.

6. Na JH, Sung KR, Lee Y. Factors associated with the signal strengths obtained by spectral domain optical coherence tomography. Korean J Ophthalmol. 2012;26:169-73.

7. Esmaeelpour M, Povazay B, Hermann B, Hofer B, Kajic V, Kapoor K, et al. Three-dimensional 1060-nm OCT: choroidal thickness maps in normal subjects and improved posterior segment visualization in cataract patients. Invest Ophthalmol Vis Sci. 2010;51:5260-6.

8. Kim NR, Lee H, Lee ES, Kim JH, Hong S, Je Seong G, et al. Influence of cataract on time domain and spectral domain optical coherence tomography retinal nerve fiber layer measurements. J Glaucoma. 2012;21:116-22.

9. Kim JH, Kim NR, Lee ES, Rho S, Kang SY, Kim CY. Influence of blue light-filtering intraocular lenses on retinal nerve fiber layer measurements by spectral-domain optical coherence tomography. Curr Eye Res. 2011;36:937-42.

10. Mwanza JC, Bhorade AM, Sekhon N, Mc Soley JJ, Yoo SH, Feuer WJ, et al. Effect of cataract and its removal on signal strength and peripapillary retinal nerve fiber layer optical coherence tomography measurements. J Glaucoma. 2011; 20:37-43.

11. Sanchez-Cano A, Pablo LE, Larrosa JM, Polo V. The effect of phacoemulsification cataract surgery on polarimetry and tomography measurements for glaucoma diagnosis. J Glaucoma. 2010;19:468-74.

12. El-Ashry M, Appaswamy S, Deokule S, Pagliarini S. The effect of phacoemulsification cataract surgery on the measurement of 
retinal nerve fiber layer thickness using optical coherence tomography. Curr Eye Res. 2006;31:409-13.

13. García-Bella J, Martínez de la Casa JM, Talavero-González P, Fernández-Vigo JI, Valcarce Rial L, García-Feijóo J Variations in retinal nerve fiber layer measurements on optical coherence tomography after implantation of trifocal intraocular lens. Eur $\mathbf{J}$ Ophthalmol 20187;28:32-5.
14. Dias-Santos A, Costa L, Lemos V, Anjos R, Vicente A, Ferreira J, et al. The impact of multifocal intraocular lens in retinal imaging with optical coherence tomography. Int Ophthalmol. 2015;35:43-7.

15. Skiadaresi E, McAlinden C, Ravalico G, Moore J. Optical coherence tomography measurements with the LENTIS Mplus multifocal intraocular lens. Graefes Arch Clin Exp Ophthalmol. 2012;250:1395-8. 